\title{
The Application of Excel in Highway Vertical Curve
}

\author{
Shan Li \\ Liaoning Technical University, Liaoning Fuxin 123000 \\ Email:846516106@qq.com
}

\author{
Li-Hong Shi \\ Chinese Academy of Surveying and Mapping, Beijing \\ Email:846516106@qq.com
}

\begin{abstract}
When we are layouting field route, the calculation of designed elevation in vertical and horizontal section is big workload and very tedious. So using Excel which has powerful data processing function and convenient operation of the form to complete a large number of height calculation in vertical and horizontal section is very convenient and practical. The paper discussed about the elevation's calculation principle of middle and side stakes in vertical curve, and mainly studied the calculation method of superelevation transition section's elevation on the winding mountain road which was accomplished by Visual Basic for Application (VBA). Then we transform the result to the required format which can transfer to the total station, so the result could layout quickly and accurately at the scene. In short, using Excel to calculate vertical curve elevation is very simple and practical. It will not only greatly improve working efficiency, but also reduce the chance of errors.
\end{abstract}

Keywords-vertical curve; elevation; superelevation; visual basic for application

\section{INTRODUCTION}

In the reforming period of society the urbanization is inevitable, and the highway is the hub of all cities, so it is very important. Vertical curve is a necessary line form in highway design and construction. Therefore studying a fast method for calculating elevation of middle and side stakes in vertical curve at different points has the very vital significance. In the previous days, we adopted the calculator to calculate the designed elevation point by point in highway vertical curve calculation, but the efficiency and fallibility of this method is very low. AutoCAD visual-lisp and Excel VBA programming language can both complete the calculation of vertical curve. With the two new methods we need not input point by point but calculate a series of points' elevation. So using Excel VBA to handle complicated elevation calculation of vertical curve is very simple and practical.

\section{VERTICAL CURVE}

In the design of road engineering, in order to adapt to the needs of terrain changes and drainage, it's necessary to set longitudinal slope. But when two adjacent longitudinal slopes intersect, there will be a grade-changing point. Vehicles will bump down when passing through this point, and driving smooth is damaged [1]. Besides slope-changing point will also obstruct the driver's view and affect driving safety. In order to reduce this effect, it is necessary to set a curve instead of the original polyline at the slope-changing point, the curve is called a vertical curve.

The purpose of vertical curve calculation is to determine subgrade design elevation at specified stake numbers design longitudinal grade, as shown in figure 1.

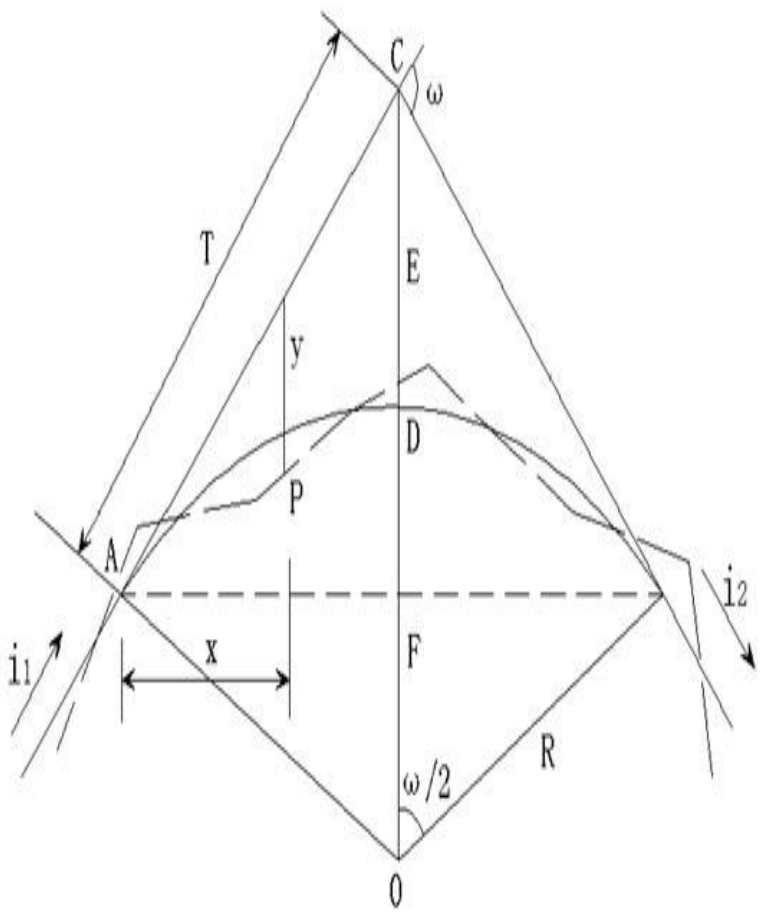

Figure 1. Vertical curve.

The vertical curve elevation calculation steps are as follows [2]:

(1) curve factors

$\alpha=a b s\left(i_{1}-i_{2}\right)$

$T=R * \alpha / 2$

$L=R * \alpha$

$E=T^{\wedge} / R / 2$

Type: $i \_$designed longitudinal gradient of vertical curve

$R$ _radius of vertical curve

$\alpha$ 
$T$ _ tangent length

$L$ _ curve length

$E$ - apex distance

(2) the starting and end points' mileage

$q d l c=b p d l c-T$

$z d l c=b p d l c+T$

Type: $q d l c$ — the starting points' mileage

bpdlc — the end points' mileage

$z d l c$ the grade-changing points' mileage

(3) any point's tangent elevation and correction value on the vertical curve

$$
\begin{aligned}
& \text { Tgc }=\text { bpdgc } \pm(T-x) * i \\
& y=x 2 / 2 / R \\
& C g c=\operatorname{Tg} c-y
\end{aligned}
$$

Type: $\operatorname{Tgc} —$ tangent elevation

bpdgc_orade-changing point elevation

$\mathrm{CgC} \_$curve elevation

$y$ correction value

\section{SUPERELEVATION}

\section{A. The Definition}

The train can produce centrifugal force when running on the curve, and the size of centrifugal force depends on the train weight, driving speed and the radius of circular curve [3]. Because of the influence of centrifugal force, the curve outer rail load increases suddenly and the inner rail load pressure decreases accordingly. When it exceeds a certain limited value, the train has the danger of derailing and overturning. In order to offset the adverse impact of the centrifugal force, railway adopts elevation of outer rail method on the curve part [4], which raises the outer rail to a certain value, makes the vehicle inclined to the inside of the curve, balances the action of centrifugal force, and ensures the safe operation of the train. Moreover due to the structure of the vehicle structure, it is necessary to widen the inner rail.

Generally there are three ways of decreasing transverse force: increasing the curve radius, slowing down driving speed and increasing the transverse slope tilted to the inside [5]. But increasing curve radius is difficult and slowing down driving speed is not recommended in the design, so the effect of setting superelevation is the best and the cost is lowest. Superelevation cross-section diagram is shown in figure 2 .

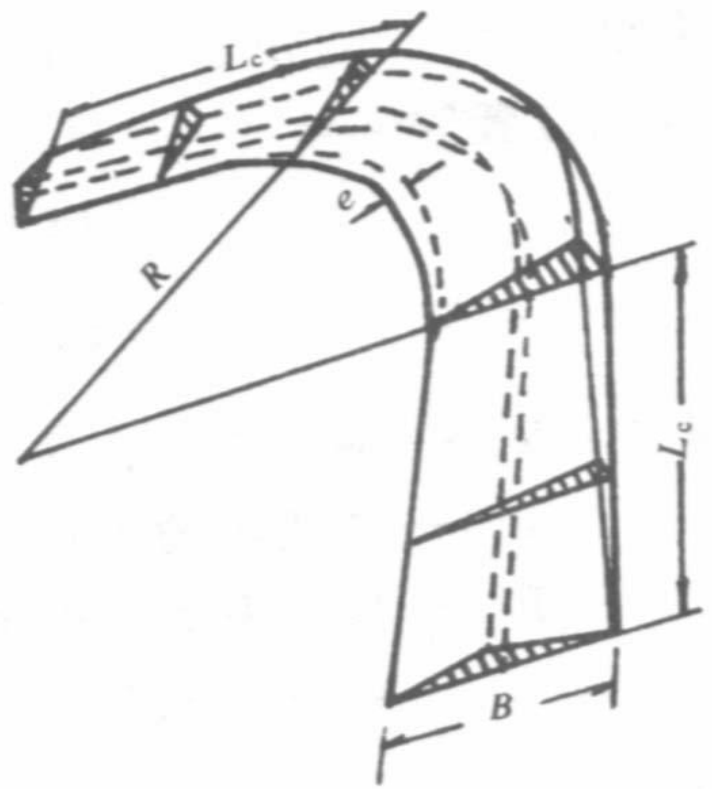

Figure 2. Cross-section diagram of superelevation

\section{B. Forming process}

The process of generating superelevation is divided into four stages [6]:

1) Preparation Stage: within the range of $1 \sim 2 \mathrm{~m}$ before getting into superelevation transition curve zone, the road should be lift its shoulder's transverse gradients up to the same as the road surface, namely the subgrade top turns into simple bidirectional transverse gradients.

2) Double way gradient stage: in superelevation transition curve zone, keeping the inside road motionless, and the outside road rotates about midline up to inside road's transverse gradients, the length of the process is called the length of double way gradient length. According to the requirement of superelevation runoff, a change in distance to transition curve zone's starting point is directly proportional to a change in crown slope.

3) Rotating stage: when the outside pavement becomes the one-way introversive transverse slope same as that of inside road, keeping the inside road motionless, then the road rotates about inner edge up to superelevation transverse slope and ends up at terminal point of transition curve zone.

4) Full superelevation stage

\section{Calculation methods}

Superelevation value is elevation difference between calculation points of subgrade design elevation, midline and road edge and shoulder edge's elevation after setting superelevation. The number of the method in superelevation transition is very big, but this paper mainly introduces rotating around the edges in the line of superelevation transition way [7]. If there is no special note, all is the way of transition. 


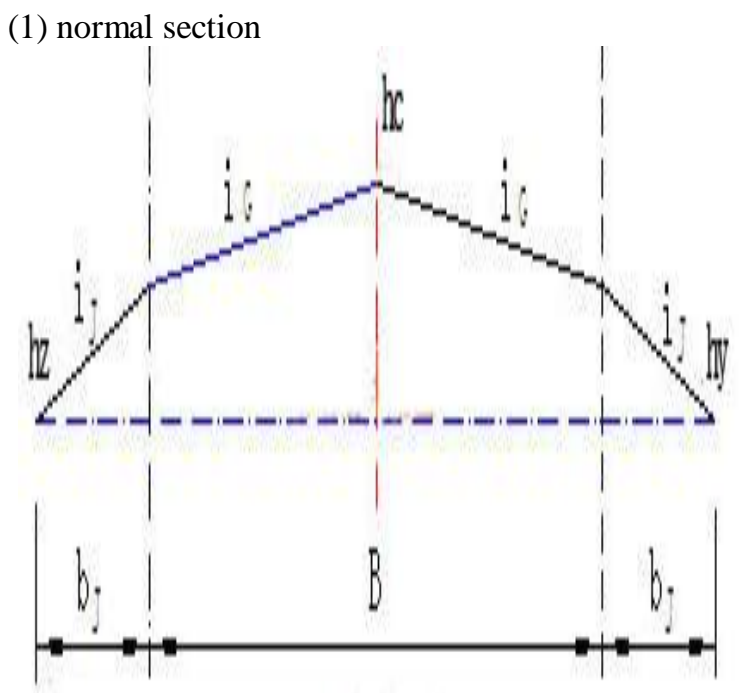

Figure 3. Diagram of normal section

$h c=b_{j} * i_{j}+B * i_{g} / 2$

$h y=b_{j} * i_{j}$

$h z=0$

Type: hc, hz, hy_ the superelevation of the road midline, pavement and shoulder respectively

$b_{j}, B \longrightarrow$ the width of shoulder and pavement respectively

$i_{j} \longrightarrow$ the transverse gradient of shoulder

$i_{g}$ - the transverse gradient of crown

(2) initial section

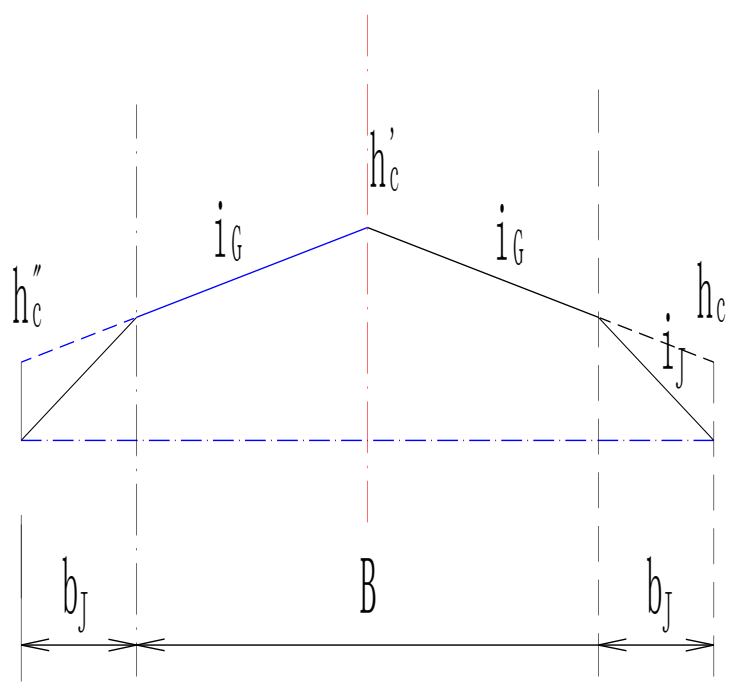

Figure 4. Diagram of initial section

$$
\begin{aligned}
& h_{c}{ }^{\prime}=b_{j} * i_{j}+B * i_{g} / 2 \\
& h_{c}=h_{c}{ }^{\prime}=b_{j} *\left(i_{j}-i_{g}\right)
\end{aligned}
$$

Type: $h^{\prime}, h^{\prime}$ ', $h$ - the superelevation of the road midline, inside edge line and outside edge line respectively

(3) double way gradient section

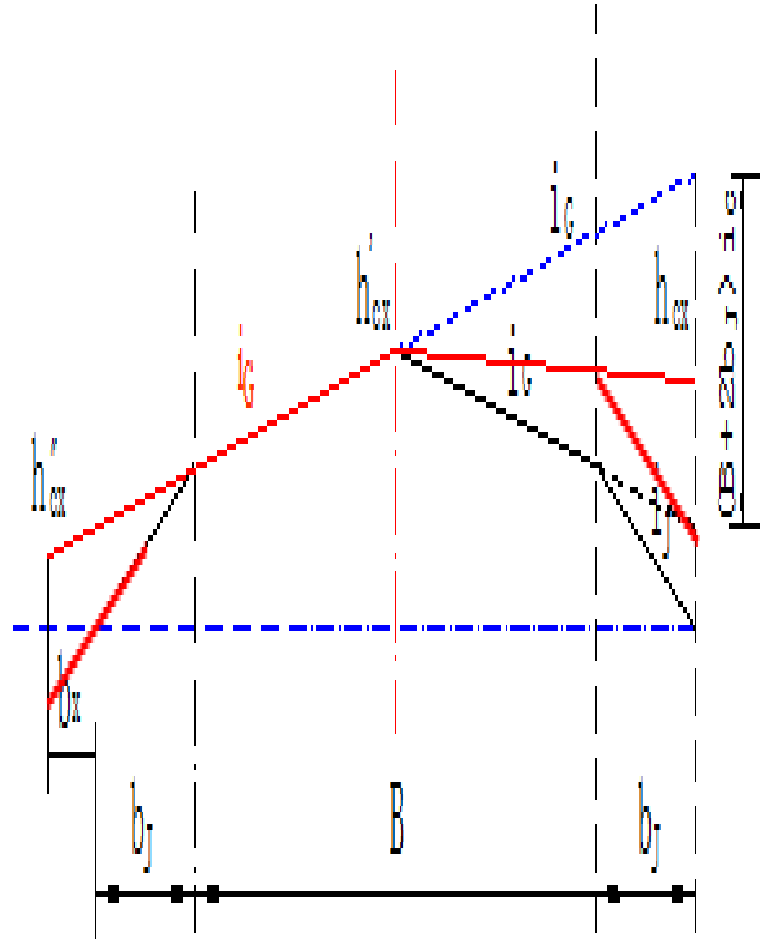

Figure5. Diagram of double way gradient section.

$$
\begin{aligned}
& x_{0}=i_{g} * l c / i_{b} \\
& h_{c x}=b_{j} *\left(i_{j}-i_{g}\right)+\frac{x}{x_{0}} *\left(B+2 * b_{j}\right) * i_{g} \\
& h_{c x},=b_{j} * i_{j}+B * i_{g} / 2 \\
& h_{c x},=b_{j} * i_{j}-\left(b_{j}+b_{x}\right) * i_{g}
\end{aligned}
$$

Type: $h_{c X}{ }^{\prime}, h_{c X}{ }^{\prime}, h_{c X} \longrightarrow$ the superelevation of the road midline, inside edge line and outside edge line respectively

$x_{0}$ - the length of double way gradient section

$i_{b}$ - the transverse gradient of superelevation

$b_{X} \longrightarrow$ the widening value at any point

(4) rotating section 


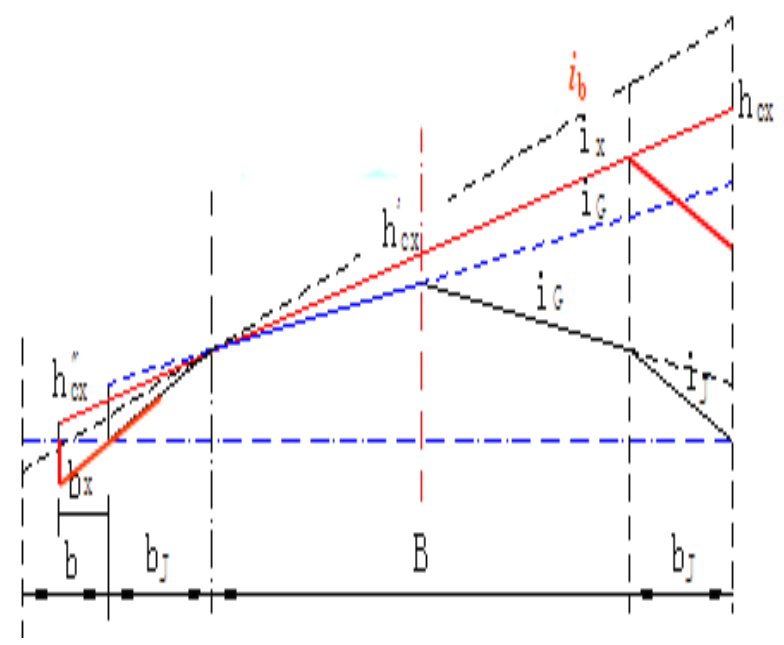

Figure 6. Diagram of rotating section.

$i_{x}=x / 1 c * i_{b}$

$h_{c x}=b_{j} * i_{j}+\left(B+b_{j}\right) * i_{x}$

$h_{c X}{ }^{\prime}=b_{j} * i_{j}+B * i_{x} / 2$

$h_{c X}{ }^{\prime}{ }^{\prime}=b_{j} * i_{j}-\left(b_{j}+b_{x}\right) * i_{X}$

Type: $1 c$ the length of the transition curve $i_{X} \longrightarrow$ the transverse gradient of rotating section

(5) full superelevation section

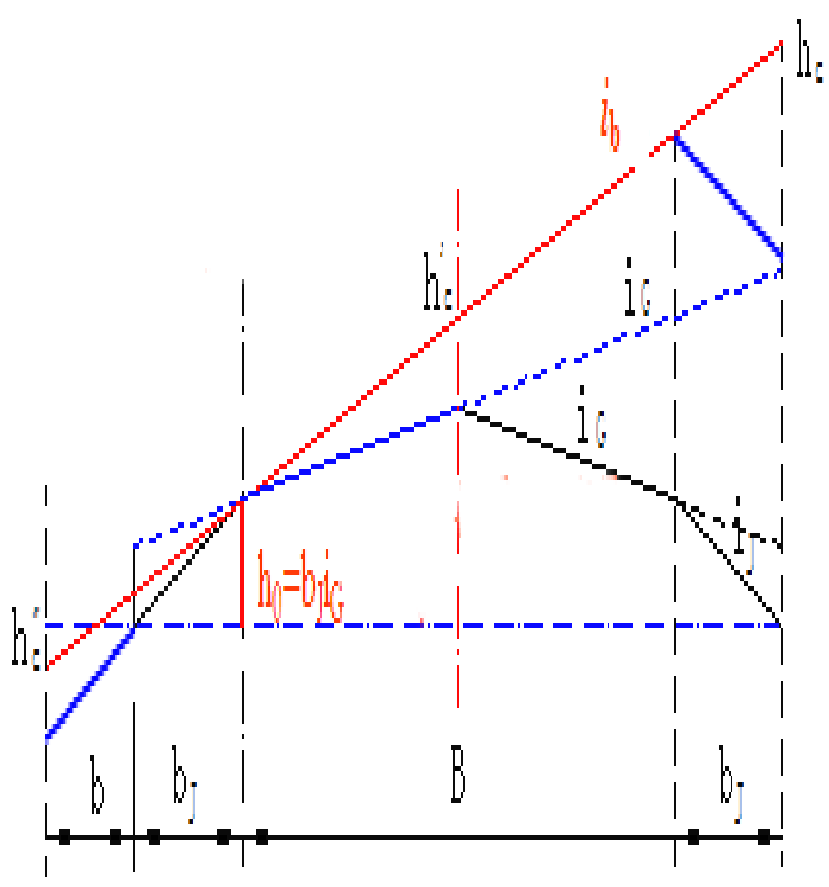

Figure7. Diagram of full superelevation section.

$$
\begin{aligned}
& h_{c x}=b_{j} * i_{j}+\left(B+b_{j}\right) * i_{b} \\
& h_{c X}^{\prime}=b_{j} * i_{j}+B * i_{b} / 2 \\
& h_{c X}{ }^{\prime}=b_{j} * i_{j}-\left(b_{j}+b\right) * i_{b}
\end{aligned}
$$

Type: $b-$ the widening value

\section{ARITHMETIC EXAMPLE}

\section{A. Data Sources and Analysis}

In this example the initial data is shown in table 1 below, suppose that midpoint of vertical curve and midpoint of plane curve is overlapped, so accordingly mileage of grade-changing point is the same as that of midpoint of plane curve, which equals to two different slopes meet in grade-changing point on the profile of winding mountain road.

TABLE I. INITIAL DATA

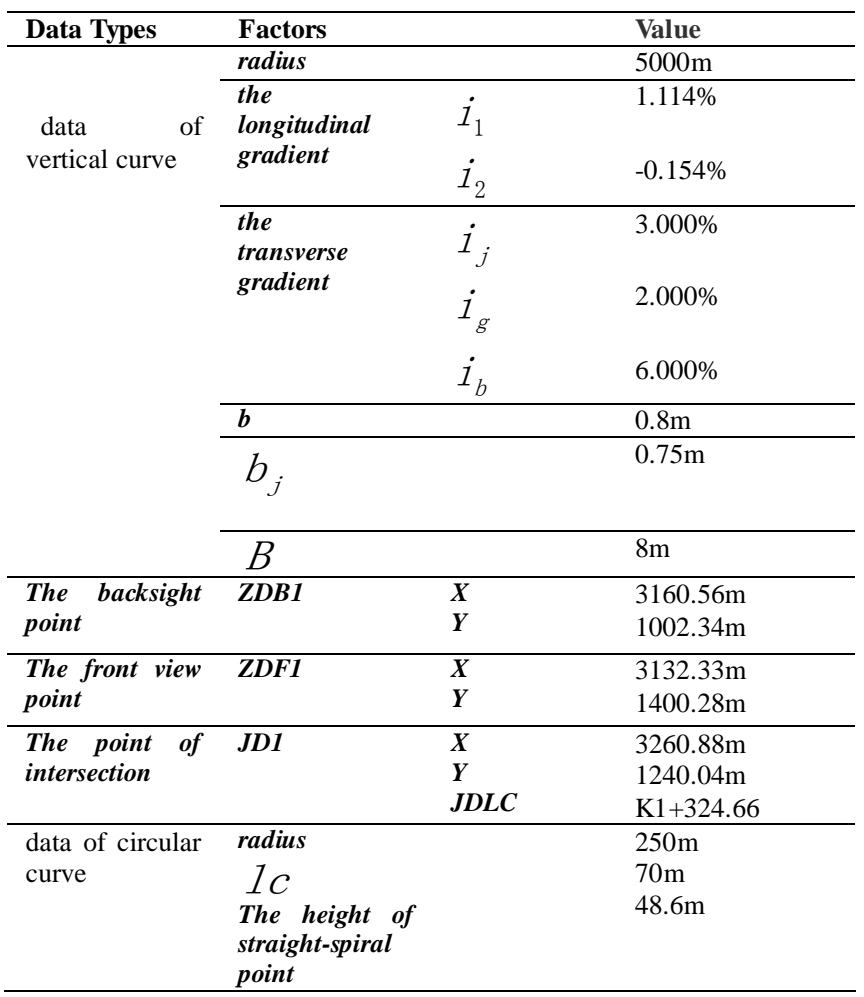

\section{B. Building Model}

According to the data determines the main points mileage on the plane curve which include coordinates and mileage data of forward-looking intersection point, intersection point and back intersection point, radius data of circular curve and length data of transition curve. Then using the straight-spiral point elevation calculates grade-changing point elevation [8]. Finally superelevation are computed by the road shoulder width and transverse slope [9]. The calculation results are shown in table 2 below. 
TABLE II. THE RESULT TABLE

\begin{tabular}{|c|c|c|c|c|c|c|c|c|c|}
\hline $\begin{array}{l}\text { stake } \\
\text { numbers }\end{array}$ & $\begin{array}{l}\text { designed } \\
\text { elevation }\end{array}$ & $b_{x}$ & $i_{X}$ & $h_{c X}{ }^{\prime}$ & $h_{c X}{ }^{\prime}$, & $h_{c X}$ & $H_{c X}{ }^{\prime}$ & $H_{c x}{ }^{\prime}$, & $H_{c X}$ \\
\hline $\mathrm{K} 1+140.110$ & 48.6000 & 0.0000 & $0.000 \%$ & 0.1 & 0.01 & 0.01 & 48.7000 & 48.6100 & 48.6100 \\
\hline $\mathrm{K} 1+150.110$ & 48.7114 & 0.1143 & $0.857 \%$ & 0.1 & 0.01 & 0.09 & 48.8114 & 48.7214 & 48.8014 \\
\hline $\mathrm{K} 1+160.110$ & 48.8228 & 0.2286 & $1.714 \%$ & 0.1 & 0 & 0.17 & 48.9228 & 48.8228 & 48.9928 \\
\hline $\mathrm{K} 1+170.110$ & 48.9342 & 0.3429 & $2.571 \%$ & 0.13 & -0.01 & 0.25 & 49.0642 & 48.9242 & 49.1842 \\
\hline $\mathrm{K} 1+180.110$ & 49.0456 & 0.4571 & $3.429 \%$ & 0.16 & -0.02 & 0.32 & 49.2056 & 49.0256 & 49.3656 \\
\hline $\mathrm{K} 1+190.110$ & 49.1570 & 0.5714 & $4.286 \%$ & 0.19 & -0.03 & 0.4 & 49.3470 & 49.1270 & 49.5570 \\
\hline $\mathrm{K} 1+200.110$ & 49.2684 & 0.6857 & $5.143 \%$ & 0.23 & -0.05 & 0.47 & 49.4984 & 49.2184 & 49.7384 \\
\hline $\mathrm{K} 1+210.110$ & 49.3798 & 0.8000 & $6.000 \%$ & 0.26 & -0.07 & 0.55 & 49.6398 & 49.3098 & 49.9289 \\
\hline $\mathrm{K} 1+248.977$ & 49.8128 & 0.8000 & $6.000 \%$ & 0.26 & -0.07 & 0.55 & 50.0728 & 49.7428 & 50.3628 \\
\hline $\mathrm{K} 1+268.977$ & 50.0356 & 0.8000 & $6.000 \%$ & 0.26 & -0.07 & 0.55 & 50.2956 & 49.9656 & 50.5856 \\
\hline $\mathrm{K} 1+288.977$ & 50.2584 & 0.8000 & $6.000 \%$ & 0.26 & -0.07 & 0.55 & 50.5184 & 50.1884 & 50.8084 \\
\hline $\mathrm{K} 1+308.977$ & 50.4812 & 0.8000 & $6.000 \%$ & 0.26 & -0.07 & 0.55 & 50.7412 & 50.4112 & 51.0312 \\
\hline $\mathrm{K} 1+328.977$ & 50.4575 & 0.8000 & $6.000 \%$ & 0.26 & -0.07 & 0.55 & 50.7175 & 50.3875 & 51.0075 \\
\hline $\mathrm{K} 1+348.977$ & 50.4267 & 0.8000 & $6.000 \%$ & 0.26 & -0.07 & 0.55 & 50.6867 & 50.3567 & 50.9767 \\
\hline $\mathrm{K} 1+368.977$ & 50.3959 & 0.8000 & $6.000 \%$ & 0.26 & -0.07 & 0.55 & 50.6559 & 50.3259 & 50.9459 \\
\hline $\mathrm{K} 1+388.977$ & 50.3651 & 0.8000 & $6.000 \%$ & 0.26 & -0.07 & 0.55 & 50.6251 & 50.2951 & 50.9151 \\
\hline $\mathrm{K} 1+408.977$ & 50.3343 & 0.8000 & $6.000 \%$ & 0.26 & -0.07 & 0.55 & 50.5943 & 50.2643 & 50.8843 \\
\hline $\mathrm{K} 1+408.980$ & 50.3343 & 0.8000 & $6.000 \%$ & 0.26 & -0.07 & 0.55 & 50.5943 & 50.2643 & 50.8843 \\
\hline $\mathrm{K} 1+418.980$ & 50.3422 & 0.6857 & $5.143 \%$ & 0.23 & -0.05 & 0.47 & 50.5722 & 50.2922 & 50.8122 \\
\hline $\mathrm{K} 1+428.980$ & 50.3268 & 0.5714 & $4.286 \%$ & 0.19 & -0.03 & 0.4 & 50.5168 & 50.2968 & 50.7268 \\
\hline $\mathrm{K} 1+438.980$ & 50.3114 & 0.4571 & $3.429 \%$ & 0.16 & -0.02 & 0.32 & 50.4714 & 50.2914 & 50.6314 \\
\hline $\mathrm{K} 1+448.980$ & 50.2960 & 0.3429 & $2.571 \%$ & 0.13 & -0.01 & 0.25 & 50.4260 & 50.2860 & 50.5460 \\
\hline $\mathrm{K} 1+458.980$ & 50.2806 & 0.2286 & $1.714 \%$ & 0.1 & 0 & 0.17 & 50.3806 & 50.2806 & 50.4506 \\
\hline $\mathrm{K} 1+468.980$ & 50.2652 & 0.1143 & $0.857 \%$ & 0.1 & 0.01 & 0.09 & 50.3652 & 50.2752 & 50.3552 \\
\hline $\mathrm{K} 1+478.980$ & 50.2498 & 0.0000 & $0.000 \%$ & 0.1 & 0.01 & 0.01 & 50.3498 & 50.2598 & 50.2598 \\
\hline
\end{tabular}

\section{CONCLUSION}

In the design of highway profile, it is necessary to calculate a lot of the designed elevation of middle and side stakes in vertical curve, especially the design elevation at any point. Calculation workload with manual is great and error-prone, so we can use Excel programming to solve this problem. Using Excel powerful editing and computing functions, we can input mileage and elevation of the change slope points and radius into the designed program, and calculate the designed elevation of middle and side stakes which can save time and money, and improve work efficiency.

In a word, the paper introduced the principle method of calculating vertical curve design elevation superelevation, using the Excel VBA [10] to solve a lot of tedious calculation problem. With examples we demonstrated that calculating vertical curve and elevation by Excel is very simple and practical.

\section{REFERENCES}

[1] GuXiaolie,BaoFeng,ChengXiaojun. Surveying[M]. Shanghai: Tongji University Press.2006.8:353, 386-387.

[2] SunYuting.Study on Application of High Superelevation[J]. Journal of Guizhou University of Technology.2007.4(36):97-99.

[3] ZhangYue,WuWenqing. The Calculation Method of Superelevation Transition on Highway Plane Curve [J]. Highways \& Transportation in Inner Mongolia.2001.1:12-13.

[4] ChenYoujun. The Calculation and Application of Highway Curve Superelevation[J].Communications Science and Technology Heilongjiang. 2008.4:43-45.

[5] GuoHailun,WangDangqiang. Batch Computing of Vertical Curve in Setting Out for Expressway Based on VB Programming[J]. Yangtze River.2012,10(43): 36-38.

[6] ZhouYan,ZhangHuaying,SongJunchao. Application of VB Conbined with EXCEL in the Height Conputation of Vertical Alignment Design[J]. Journal of Jiamusi University (Natural Science Edition). 2009,3(27): 408-410.

[7] XuGuangxiang, WangShuliang. The Height Computation Research of the Vertical Curve and Superelevation Gradient Section in Highway[J]. Shanxi Architecture.2006,12(32): 364-365.

[8] JiangYanhui,XuDexing. Plane Curve Excedding High and Gentle Curve[J]. Forestry Science and Technology Information.2001.1: 59-60.

[9] The People's Republic of China. Highway Engineering Technique Standards. Beijing:China Communication Press, 1997.

[10] LiFang. Excel VBA efficient office super handbook[M].Beijing: China Machine Press.2011.3:211-218. 